\title{
Evolution of damped Lyman-alpha galaxies from hierarchical structure formation models
}

\author{
Katsuya Okoshi ${ }^{1}$ and Masahiro Nagashima ${ }^{2}$ \\ ${ }^{1}$ National Astronomical Observatory, Mitaka, Tokyo 181-8588, Japan \\ email: okoshi.katsuya@nao.ac.jp \\ ${ }^{2}$ Department of Physics, Kyoto University, Sakyo-ku, Kyoto 606-8502, Japan
}

\begin{abstract}
We investigate the evolution of damped Ly $\alpha$ absorption systems (DLA) from hierarchical structure formation models by exploring the basic properties of DLA host-galaxies (DLA galaxies) to clarify the connection between DLAs and galaxies. We find that DLA galaxies primarily consist of low surface brightness dwarf galaxies. For example, at redshift $z \leqslant 1$, DLA galaxies have typical radius $3 \mathrm{kpc}$, surface brightness $22-27 \mathrm{mag}$ arc-sec ${ }^{-2}$, and star formation rate $10^{-2} \mathrm{M}_{\odot} / \mathrm{yr}$. We discuss selection effects in the detection in emission caused by compactness and brightness. Finally, we explore the radio properties at $z=0$ by comparing the observational results from blind radio surveys.
\end{abstract}

\section{Introduction}

The numerous absorption lines found in quasar spectra are one of the few observational opportunities that provide us fruitful information on the physical state of the evolving Universe. DLA systems, which give rise to damped Ly $\alpha$ absorption lines, provide exceptional insights into exploring galaxy formation in an evolutionary stage.

Semi-analytic modelling has been applied with a view to deciphering the clues to the formation process of galaxies in the hierarchical clustering scenario. This approach takes into account the merging histories of dark halos based on CDM models, and has successfully provided galaxy formation models for explaining the observational properties of galaxies (e.g. luminosity functions). It has some advantages over numerical hydrodynamical simulations. For example, under simple recipes for star formation or supernova feedback processes, it does not suffer from resolution limitations in numerical hydrodynamic simulations. This advantage allows us to study the evolutionary processes of compact objects that are difficult to resolve by numerical simulations. Recently, some observational results suggest that dwarf galaxies could contribute to the population of DLAs. Therefore, it is valuable to compare the observed properties of DLA galaxies with those predicted by the theoretical models as a useful test of theories of galaxy formation.

\section{Model}

We explore DLA galaxy formation and evolution in a semi-analytic modelling framework. Our model employs a Monte Carlo method for generating merger trees to depict the hierarchical growth of dark matter halos. It incorporates descriptions of gas evolution. When a halo collapses, halo gas is shock-heated. Then, a galactic disk forms in the cold gas which cools quickly in from the hot gas. This model follows the chemical evolution of gas and stars under prescriptions for star formation and supernova feedback processes. The star formation rate (SFR) is given by $\dot{M}_{*}=M_{\text {cold }} / \tau_{*}$ where $M_{*}$ and $M_{\text {cold }}$ are the masses in stars and cold gas, respectively. $\tau_{*}$ is the timescale of star formation that is 
set by matching the model prediction for the cold gas mass fraction of spiral galaxies to the one observed, because these directly determine the gas consumption rate. The reheating rate by SNe is $\dot{M}_{\text {reheat }}=\beta \dot{M}_{*}$ when $\beta \propto V_{\text {circ }}^{-2.5}$ because feedback processes more seriously affect less massive galaxies. For DLAs, we assume the basic quantities under the following assumptions. The radial distribution of the HI column density follows an exponential profile with the effective radius of a face-on gaseous disk. The effective radius is given by the specific angular momentum conservation of cooling hot gas at $z=0$ (the dimensionless spin parameter has a log-normal distribution). The central column density $N_{0}$ is given by $N_{0}=M_{\text {cold }} /\left(2 \pi \mu m_{\mathrm{H}} r_{\mathrm{e}}^{2}\right)$, where $m_{\mathrm{H}}$ is the mass of a hydrogen atom and $\mu$ is the mean molecular weight. The size of a DLA system is defined by the radius $R$ at which $N_{\mathrm{HI}}=10^{20} \mathrm{~cm}^{-2}$. For each system, we take the column density averaged over radius within $R$. The detail prescriptions for modelling individual physical processes are described in Okoshi et al. (2004). This model reproduces well many aspects of the observed properties of galaxies such as luminosity functions, cold gas mass fractions, disk sizes, and faint galaxy number counts (Nagashima et al. 2001). We apply this model to a $\Lambda$ CDM cosmology $\left(\Omega_{0}, \Omega_{\Lambda}, h\right)=(0.3,0.7,0.7)$.

\section{Results}

Our model has the ability to reproduce various properties not only of galaxies (Nagashima et al. 2001), but also of DLA systems, particularly the observed distributions in the HI column density and the metallicity evolution (Okoshi et al. 2004). Here, we present our results for low-redshift DLA galaxies (Okoshi \& Nagashima 2005). At low redshift $(z \leqslant 1)$, DLA galaxies have been studied from both direct photometric images and spectroscopic follow-ups (e.g. Rao et al. 2003, and references therein). The observations (e.g. luminosities, impact parameters) offer clues to revealing the physical relation between DLAs and galaxies. In Fig. 1 (Left), we show the results of low-redshift DLA galaxies in comparison to the observations. It should be noted that our model incorporates selection effects that affect the detection of DLA galaxies caused by (1) cosmological dimming of the surface brightness and (2) the masking effect which prevents us from identifying a DLA galaxy hidden or contaminated by the point-spread function of a background quasar. We find that the observational data are well reproduced by our model that accounts for the selection biases. Our results suggest that DLA systems primarily consist of low luminosity galaxies with small impact parameters (typical radius $\sim 3 \mathrm{kpc}$, surface brightness from 22 to $27 \mathrm{mag}$ arc-sec ${ }^{-2}$ ). Because of the small disks, the masking effect affects the distributions of DLA properties seriously in our results. For example, the rate of DLA galaxies missed because of the masking effect reaches $60-90 \%$ in the sample at redshifts $0 \leqslant z \leqslant 1$ when the angular size limit is as small as 1 arc-sec.

Finally, we calculate the radio properties provided by blind surveys that directly detect HI fluxes from HI-rich galaxies. In Fig. 1 (Right), we present our results for some relations with HI mass of DLA galaxies. In particular, we find a tight correlation between HI mass and cross section of DLA galaxies, $\sigma \propto M_{\mathrm{HI}}^{\alpha}$ with $\alpha=0.97 \pm 0.01$. This is entirely consistent with the properties of HI-selected galaxies obtained by a blind radio survey: the Arecibo Dual-Beam Survey (Rosenberg \& Schneider 2003).

\section{Conclusions}

We have investigated damped Ly $\alpha$ absorbing galaxies by our galaxy formation model (Okoshi et al. 2004) in the hierarchical structure formation scenario to explore the photometric and radio properties comprehensively enough to compare with the currently available observations. The main conclusions are the following: 

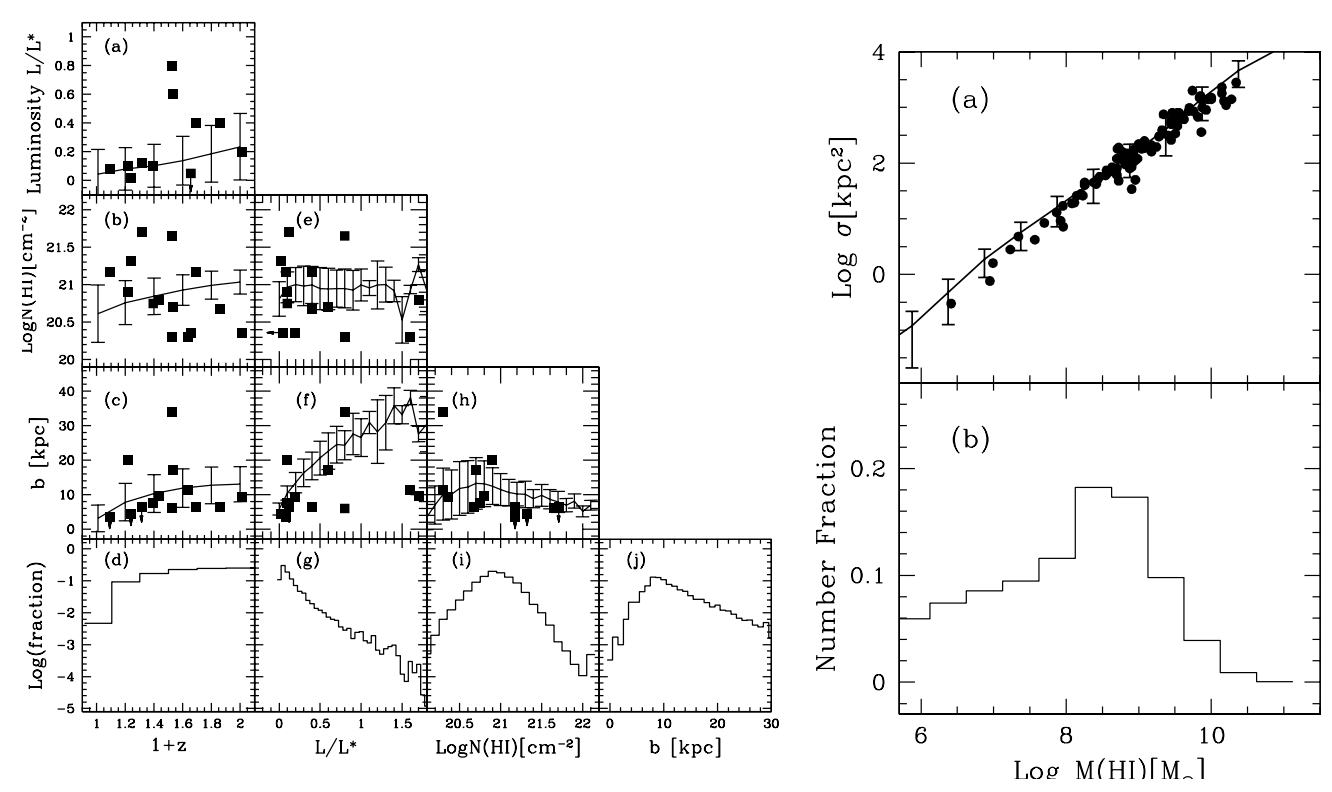

Figure 1. Left panel: fundamental properties of DLA galaxies at redshifts $0 \leqslant z \leqslant 1$ when the detection limits of central surface brightness $\mu_{\mathrm{B}}=25\left[\mathrm{mag} \operatorname{arc}_{-\mathrm{sec}^{-2}}\right]$ and angular size $\theta=1$ [arc-sec]. Error bars with the averages indicate $1 \sigma$ errors. The squares present observational data (More details are presented in Rao et al. 2003). Right panel: relations with HI mass of DLA galaxies at $0 \leqslant z \leqslant 1$. (a) The cross section vs. HI mass. The average cross sections at $z=0$ are plotted as the solid line. Error bars with the averages indicate $1 \sigma$ errors. The filled circles represent observational data from blind $21 \mathrm{~cm}$ surveys (Rosenberg \& Schneider 2003). (b) Number fraction of DLA galaxies as a function of HI mass at $0 \leqslant z \leqslant 1$.

(a) DLA galaxies primarily consist of low surface brightness (LSB) dwarf galaxies; e.g. the typical radius $\sim 3 \mathrm{kpc}$, the mean surface brightnesses are $\sim 22-27$ mag arc-sec $^{-2}$ at redshifts $z \leqslant 1$.

(b) Our results agree better with the observations taking into account two selection effects caused by (1) the low surface brightness $\left(\mu_{\mathrm{th}}=25 \mathrm{mag}\right.$ arc-sec $\left.{ }^{-2}\right)$ and (2) the masking effect $\left(\theta_{\mathrm{th}}=1^{\prime \prime}\right)$. The rate of DLA galaxies missed by the selection bias reaches $60-90 \%$ if low-luminosity galaxies with small impact parameters significantly contribute to the population of DLA systems.

(c) A tight relation between the HI mass and the cross-section is confirmed in DLA systems. HI-rich galaxies with $10^{9} \mathrm{M}_{\odot}$ mainly contribute to the population at $z \leqslant 1$. These results are entirely consistent with the properties of HI-selected galaxies in a radio survey.

\section{References}

Nagashima, M., Totani, T., Gouda, N., Yoshii, Y., 2001, ApJ, 557, 505

Okoshi, K., Nagashima, M., 2005, ApJ, 623, 99

Okoshi, K., Nagashima, M., Gouda, N., Yoshioka S., 2004, ApJ, 603, 12

Rao, S. M., et al., 2003, ApJ, 595, 94

Rosenberg, J. L., Schneider, S. E., 2003, ApJ, 585, 256 